\title{
Study by optical techniques of the dependence of aggregation parameters of human red blood cells on their deformability
}

\author{
Anastasia I. Maslyanitsyna ${ }^{1 *}$, Petr B. Ermolinsky ${ }^{1}$, Andrei E. Lugovtsov ${ }^{1,2}$, \\ and Alexander V. Priezzhev ${ }^{1,2}$ \\ ${ }^{1}$ Physics faculty, Lomonosov Moscow State University, Leninskie Gory 1-2, Moscow 119991, GSP-1, Russian Federation \\ ${ }^{2}$ International Laser Centre, Lomonosov Moscow State University, Leninskie Gory 1-62, Moscow 119991, GSP-1, \\ Russian Federation
}

* e-mail: stasyazavr2000@biomedphotonics.ru

\begin{abstract}
Blood microcirculation in human body is greatly dependent on the microrheologic properties of red blood cells. The aim of this work is to identify the relationship between the deformability of these cells and their aggregation properties, both of which are the key factors for the blood flow. Laser diffractometry, diffuse light scattering and laser tweezers were implemented for in vitro measurements. Different osmolarity of plasma (150-500 mOsm/l) and concentrations of glutaraldehyde (up to $0.004 \%$ ) were used to change the deformability of healthy red blood cells in vitro. The results show that with the cells becoming more rigid some aggregation parameters (e.g. the fraction of aggregated cells) decrease, while some of them (e.g. the hydrodynamic strength of the aggregate) stay unchanged. For example, after incubation in $0.004 \%$ glutaraldehyde solution the erythrocyte deformability drops by $19 \pm 2 \%$ and this leads to a decrease by $77 \pm 4 \%$ in the aggregation index. This means that there is a connection between cell deformability and the formation of the aggregates, however the relationship is less pronounced and more complex for the disaggregation process. (C) 2020 Journal of Biomedical Photonics \& Engineering.
\end{abstract}

Keywords: blood; erythrocytes; red blood cell aggregation; deformability; laser diffractometry; laser tweezers; diffuse light scattering; glutaraldehyde; osmolarity.

Paper \#3359 received 19 May 2020; revised manuscript received 15 Jun 2020; accepted for publication 19 Jun 2020; published online 22 Jun 2020. doi: 10.18287/JBPE20.06.020305.

\section{Introduction}

The state of human organism largely depends on blood microcirculation that, in turn, depends on the microrheologic properties of red blood cells (RBCs), in particular, the RBC intrinsic properties of deformability and aggregation that some research [1] shows as interdependent.

The RBCs have the ability to be deformed in the blood flow. Usually they elongate in the direction of the flow, but also, they can change their shape dramatically in the vessels that are smaller than the size of the RBCs, for example, capillaries with a 3 to $5 \mu \mathrm{m}$ radius [2]. A considerable contribution to the deformability comes from the elasticity of the cell membrane, as well as from the hemoglobin solution inside [3]. RBC deformability plays a significant role in the blood circulation. In particular, RBC filtering in narrow circulatory pathways in the human spleen is based on their impaired deformability [4].

Another important process that influences the blood flow is the aggregation of RBCs [5]. It is a reversible process of creation of linear and more complex structures of RBCs. The aggregation happens dominantly inside large vessels [5]. However, the aggregates can become quite large themselves, and if 
not their ability to disaggregate to single cells, the blood flow would be impaired.

Socially significant diseases such as arterial hypertension, diabetes mellitus and others are associated with serious changes in the RBC deformability [6, 7]. At the same time a significant change in the aggregation parameters happens. For example, RBC aggregates in the blood of patients suffering from arterial hypertension are stronger and form faster than in the blood of healthy people [8]. Moreover, these pathologies are accompanied by an alteration in the number of RBC involved in the process of spontaneous aggregation [9]. This can be caused by many reasons: a change in the protein composition of plasma, cell membrane changes, different rigidity and age of the cells, as well as the average patient age and their medication, etc. [5].

The aim of this work is to study the effect of RBC deformability on their aggregation properties using optical methods in vitro. By establishing this relationship, one can better predict the effect of drugs such as Semax [10] on both of the discussed parameters. Also, it is a small step towards understanding the RBC aggregation process fundamentally.

\section{Materials and methods}

\subsection{Blood samples}

The blood was drawn from two healthy individuals who gave informed consent. Blood was stabilized by EDTA anticoagulant. The experiments were carried out within $3 \mathrm{~h}$ after the sample collection. The RBCs were separated from the plasma by centrifuging the sample at $170 \mathrm{~g}$ for $10 \mathrm{~min}$, then the plasma was centrifuged for 10 min at $3000 \mathrm{~g}$ to remove the buffy coat. After that, two treatments were used to change the deformability of RBC. The first one included washing the RBCs thrice with isotonic phosphate-buffered saline (PBS), incubating them in glutaraldehyde (GA) solutions in a range 0 to $0.004 \%$ for 30 minutes at room temperature (according to the protocol in Ref. [11]), washing them three times in PBS again and then adding them to plasma at $40 \%$ hematocrit. The second one included mixing the plasma with $\mathrm{PBS}$ and $\mathrm{NaCl}$ in order to achieve different osmolarities 150 to $500 \mathrm{mOsm} / 1$ and then adding RBC at $40 \%$ hematocrit. Next, three optical techniques described in more detail in the work [12] were used to study the cells in vitro.

\subsection{Laser tweezers}

A part of the measurements was carried out with laser tweezers using a highly diluted RBC suspension [13]. Two-channel optical tweezers based on a Nd:YAG laser with diode pumping $(\lambda=1064 \mathrm{~nm}, 200 \mathrm{~mW})$ allow us to manipulate single cells and measure the forces of interaction between them without a direct mechanical contact [7]. Thus, numerical values of the forces of aggregation and disaggregation on pairs of single RBCs (doublets) were obtained. The aggregation force is the minimum force necessary to prevent spontaneous aggregation and the disaggregation force is the minimum force required to separate a pair of aggregated RBCs. The measurements were performed at room temperature.

\subsection{Laser aggregometry}

Laser aggregometry was performed using the RheoScan aggregometer (Rheomeditech, Seoul, Korea) [14]. It is based on diffuse light scattering and is applied to whole blood samples in order to retrieve a number of the RBC aggregation properties. By analyzing the scattered light intensity as a function of time during the process of RBC spontaneous aggregation we can evaluate the aggregation index (AI), which characterizes the ratio of aggregated cells during the first $10 \mathrm{sec}$ of the aggregation process [14]. Besides that, the critical shear stress (CSS) that characterizes the balance of aggregation and disaggregation processes was measured. In order to do it, the blood flow conditions were created in vitro with varying shear stress, and the light scattered backwards was analyzed. The measurements were performed at $37^{\circ} \mathrm{C}$.

\subsection{Laser deformometry}

Laser deformometry performed with the RheoScan diffractometer was used to obtain the shear induced deformation parameters of RBCs by processing the light intensity distribution in the diffraction pattern [15]. This pattern is based on diffraction of a laser beam on a highly diluted RBC suspension in a flow channel in vitro. The dimensions of the channel are $0.2 \mathrm{~mm}$ high $\times 4.0 \mathrm{~mm}$ wide $\times 40 \mathrm{~mm}$ long. We measured the RBC deformability index (DI) that describes the average elongation of the cells by shear stress illuminated by the laser beam induced. The elongation of the cells corresponds to the elongation of the diffraction pattern. Different shear stresses from $20 \mathrm{~Pa}$ to $0.5 \mathrm{~Pa}$ are applied to the $\mathrm{RBC}$ suspension in order to change the shear stress and, consequently, the elongation of the cells. Shear stress is calculated automatically assuming a parabolic velocity profile [11]. The measurements were performed at $37^{\circ} \mathrm{C}$.

\subsection{Statistical analysis}

This study was conducted on the blood of 2 healthy donors. The values of AI, DI and the forces of RBC interaction were measured 5 times for the same sample. The results were then averaged and the standard deviations from the mean values were calculated.

\section{Results and discussion}

\subsection{RBC deformability changes}

The RBC deformability changes are presented in the Fig. 1. Firstly, results in the Fig. 1a show a decrease in the deformability index with the increase of GA 
concentration that corresponds to the conclusions from the study [16]. The deformability of all GA treated samples is lower than that of the control sample. The deformability index at the highest shear stress of $20 \mathrm{~Pa}$ of the sample with maximum GA concentration $(0.004 \%)$ is $16 \pm 2 \%$ lower than the control. For shear stresses less than $6 \mathrm{~Pa}$ the relationship is not that clear. This can happen because the viscosity of the cytoplasm inside of the cell plays a role in the deformability at sufficiently high shear stress. As discussed in Ref. [17] GA cross-links the proteins inside the cells. In the study [18] the authors present a dependence of RBC deformability for different GA concentrations measured with ectacytometer LORRCA. It shows a conclusive decrease of deformability in the samples treated with GA at high shear stress similar to that in Fig la.

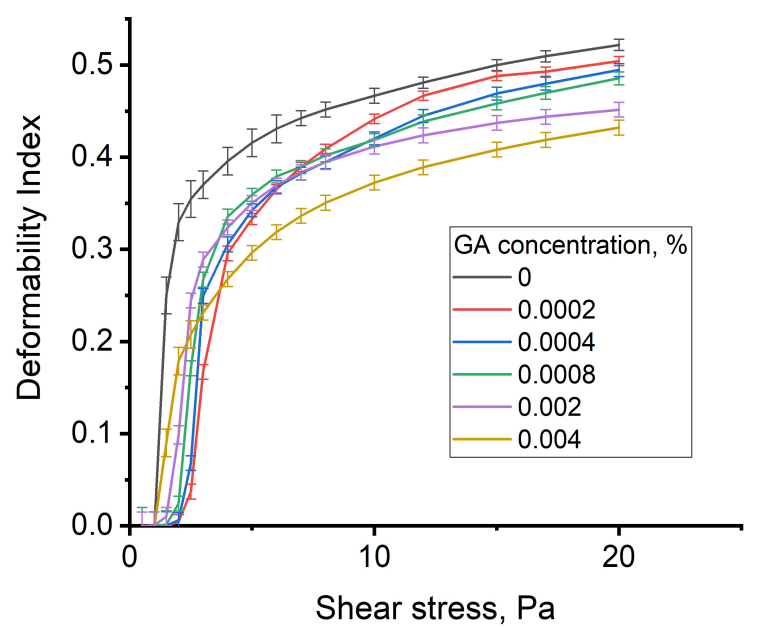

(a)

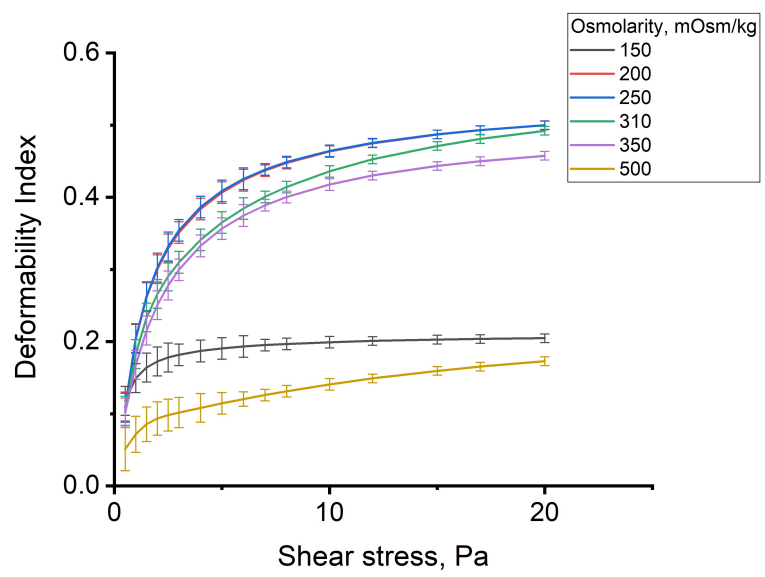

(b)

Fig. 1 The deformability index as a function of the shear stress (a) at different glutaraldehyde concentrations and (b) at different osmolarities. The values were measured 5 times for each donor. The averaged values for two donors and the standard deviations from the mean are presented.

Secondly, in a separate experiment, the osmolarity of the suspension was also shown to influence RBC deformability as seen in Fig. 1b. The normal osmolarity of plasma of two healthy volunteers was measured to be $300 \mathrm{mOsm} / \mathrm{l}$. We found that the deformability curve corresponding to the normal osmolarity level does not lie above all other curves, and even at $20 \mathrm{~Pa}$ the DI control value is similar to that of 200 and $250 \mathrm{mOsm} / 1$. However, any significant upward or downward variations of osmolarity decrease the RBC deformability dramatically. A similar dependence is described in the following work [19]. A more complicated relationship between osmolarity and deformability is discussed in Ref. [20], where the authors show that the deformability of RBC actually rises again at around $100 \mathrm{mOsm} / 1$.

\subsection{RBC aggregation changes}

The results obtained by laser aggregometry for the samples treated with GA are shown in Fig. 2. The aggregation index significantly drops for samples with low DI, which corresponds to high GA concentration. Namely, the control measurement ( $0 \%$ GA) yields DI equal to $0.522 \pm 0.006$ and $\mathrm{AI}$ equal to $39 \pm 4 \%$. For $0.004 \%$ GA concentration DI decreases to $0.426 \pm 0.006$ and AI decreases to $9 \pm 3 \%$. This means that at high GA concentrations the process of spontaneous aggregation almost stops.

The CSS parameter (Fig. 2b) remains unchanged at $160 \pm 40 \mathrm{mPa}$ for all DI levels without any significant differences due to the large deviations of its values. On the one hand, the balance of aggregation and disaggregation in flow conditions obviously depends on the aggregability of $\mathrm{RBC}$, but on the other hand, this method measures the hydrodynamic strength of the aggregates, not their number in the sample. Article [21] suggests that membrane deformability does not influence the aggregate break-up in steady uniform shear flow. Also, the difference between the deformability of interacting RBC within one sample can explain large deviations. The following computer model [22] based on the depletion theory of RBC aggregation shows that cells with different deformabilities have a reduced aggregating tendency in shear conditions.

For the samples suspended in different osmolarities the laser aggregometry method shows similar results (Fig. 3a). Lower deformability corresponds to smaller aggregation index. No statistically significant influence of the deformability on CSS was found.

However, more intriguing results are presented on Fig. 3b. The effect of osmolarity (250 to $500 \mathrm{mOsm} / \mathrm{l})$ on the forces of interaction between $\mathrm{RBC}$ in a doublet is more pronounced. The forces are presented in relative units, normalized to the aggregation force with an osmolarity of $300 \mathrm{mOsm} / 1$. This graph clearly shows a decrease in aggregation force at low deformability. At the same time, the disaggregation force also decreases, but very slightly. In the sample with low osmolarity of $250 \mathrm{mOsm} / \mathrm{l}$, the force values are not significantly different from those in the $300 \mathrm{mOsm} / 1$ sample. This shows that the disaggregation process is less responsive to changes in the deformability of RBC. 


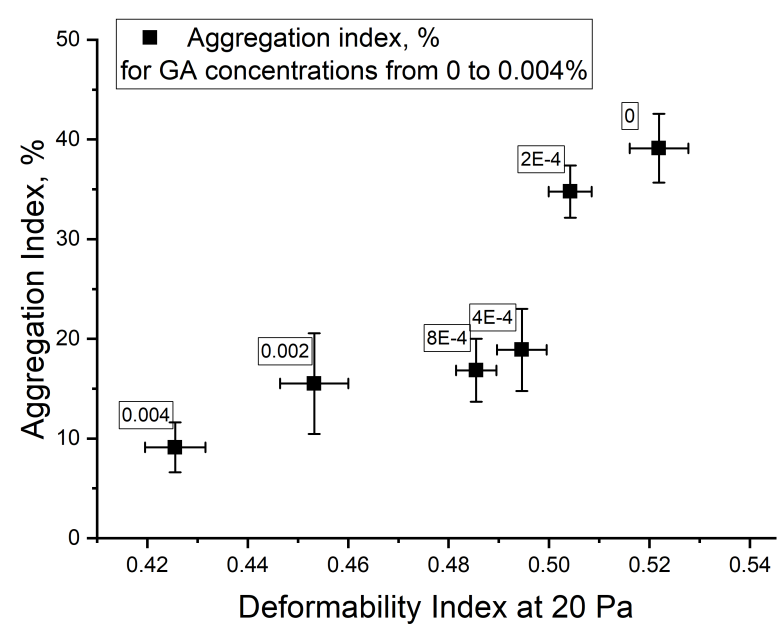

(a)

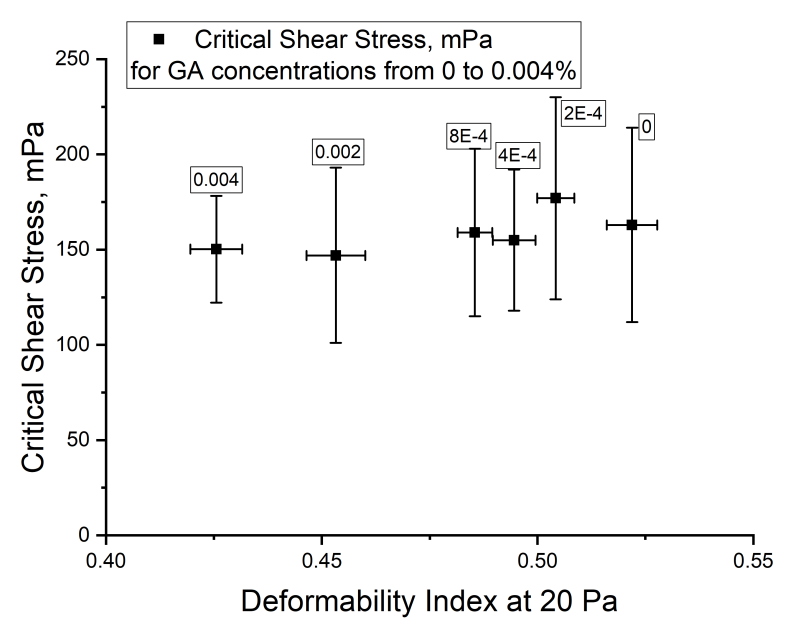

(b)

Fig. 2 (a) The aggregation index and (b) critical shear stress as functions of the deformability index for different glutaraldehyde concentrations (labeled). The values were measured 5 times for each donor. The averaged values for two donors and the standard deviations from the mean are presented.

To sum up, the parameters describing the formation of aggregates (aggregation index and force) decrease significantly for less deformable RBCs. However, the parameters describing the aggregates destruction (critical shear stress and disaggregation force) do not show a strong dependence on the deformability of RBCs. Both of these processes are complex and depend not only on the RBC properties, but also on the composition of the surrounding plasma. It was found that the hydrodynamic strength of the aggregates increases along with simultaneous reduction of RBC deformability and elevation of fibrinogen levels [23]. Research [13] performed with laser tweezers shows that the disaggregation process in large part can be described with a migrating cross-bridging mechanism of cell interaction, while the description of RBC aggregation requires additional factors. Nevertheless, further study with different methods is needed to assess the synergetic effects of protein concentration, deformability and others on aggregation of RBCs.

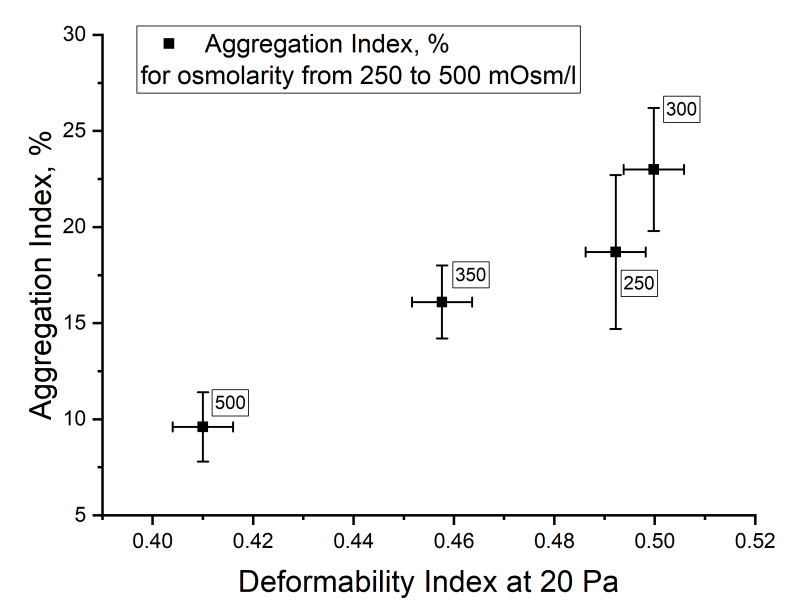

(a)

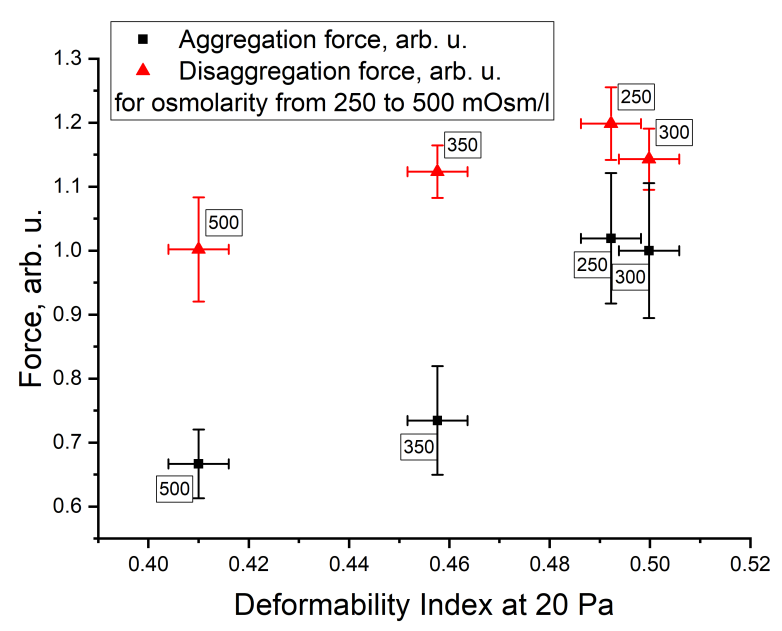

(b)

Fig. 3 (a) The aggregation index and (b) forces of aggregation and disaggregation as functions of the deformability index for different osmolarities (labeled). The values were measured 5 times for each donor. The averaged values for two donors and the standard deviations from the mean are presented.

\section{Conclusion}

Firstly, the method of laser diffractometry confirmed that with addition of glutaraldehyde and with a large change in the osmolarity of the solution, red blood cells become more rigid. Secondly, the methods of laser aggregometry and laser tweezers gave consistent results: with the decreased ability of red blood cells to deform the formation of aggregates becomes impaired. However, the critical shear stress and the disaggregation force remain mostly unchanged. This means that the RBC aggregate formation is dependent on the deformability of the membrane, while the connection to 
disaggregation is less pronounced and more complicated in nature.

These results confirm the existence of a complex relationship between the selected microrheologic parameters of blood, and it is necessary to study thoroughly this relationship and keep it in mind when diagnosing and treating various cardiovascular diseases. Of course, these findings are valid only for in vitro conditions. Further experiments are needed to correlate this work with in vivo studies.

\section{Disclosures}

All authors declare that there is no conflict of interests in this paper.

\section{Acknowledgments}

This work was supported by the Russian Foundation for Basic Research grant \#19-52-51015.

\section{References}

1. V. Leftov, S. Regirer, and N. Shadrina, Blood Rheology, Meditsina (1982) [in Russian].

2. V. V. Tuchin (Ed.), Handbook of Optical Biomedical Diagnostics, Vol. 2, second edition, SPIE PRESS, Bellingham, Washington, USA (2016).

3. N. Firsov, A. Priezzhev, N. Klimova, and A. Tyurina, "Fundamental laws of the deformational behavior of erythrocytes in shear flow," Journal of Engineering Physics and Thermophysics 79(1), 118-124 (2006).

4. H. Li, L. Lu, X. Li, P. A. Buffet, M. Dao, G. E. Karniadakis, and S. Suresh, "Mechanics of diseased red blood cells in human spleen and consequences for hereditary blood disorders," Proceedings of the National Academy of Sciences, 115(38), 9574-9579 (2018).

5. O. Baskurt, B. Neu, and H. Meiselman, Red blood cell aggregation, CRC Press (2012).

6. O. Fadyukova, A. Lugovtsov, A. Priezzhev, and V. Koshelev, "Optical study of blood rheological properties for krushinsky-molodkina strain rats with diabetes mellitus and acute disturbances of the cerebral circulation," Series Physics 17, 111-120 (2017) [in Russian].

7. P. Ermolinskiy, A. Lugovtsov, A. Maslyanitsina, A. Semenov, L. Dyachuk, and A. Priezzhev, "Interaction of erythrocytes in the process of pair aggregation in blood samples from patients with arterial hypertension and healthy donors: measurements with laser tweezers," Journal of Biomedical Photonics \& Engineering 4(3), 030303, (2018).

8. P. Ermolinskiy, A. Lugovtsov, A. Maslyanitsina, A. Semenov, L. Dyachuk, and A. Priezzhev, "In vitro assessment of microrheological properties of erythrocytes in norm and pathology with optical methods," Series on Biomechanics, 32(3), 20-25 (2018).

9. Yu. Gurfinkel, A. Lugovtsov, P. Ermolinskiy, E. Pavlikova, L. Diachuk, and A. Priezzhev, "Comparative in-vivo and in-vitro study of blood rheological properties in patients with coronary heart disease with laser-optic techniques," Proceedings of SPIE 11065, 110650 U (2019).

10. O. Fadyukova, A. Yu. Tyurina, A. E. Lugovtsov, A. V. Priezzhev, L. A. Andreeva, V. B. Koshelev, and N. F. Myasoedov, "Semax increases erythrocyte deformability in the shearing blood stream in intact rats and rats with cerebral ischemia," Doklady Biological Sciences 439, 208-211 (2011).

11. O. Baskurt, M.R. Hardeman, M. Uyuklu, P. Ulker, M. Cengiz, N. Nemeth, S. Shin, T. Alexy, and H. J. Meiselman, "Comparison of three commercially available ektacytometers with different shearing geometries," Biorheology 46(3), 251-264 (2009).

12. A. Lugovtsov, Y. I. Gurfinkel, P. B. Ermolinskiy, A. I. Maslyanitsina, L. I. Dyachuk, and A. V. Priezzhev, "Optical assessment of alterations of microrheologic and microcirculation parameters in cardiovascular diseases," Biomedical Optics Express 10(8), 3974-3986 (2019).

13. K. Lee, M. Kinnunen, M. D. Khokhlova, E. V. Lyubin, A. V. Priezzhev, I. Meglinski, and A. A. Fedyanin, “Optical tweezers study of red blood cell aggregation and disaggregation in plasma and protein solutions," Journal of Biomedical Optics 21(3), 035001 (2016).

14. S. Shin, Y. Yang, and J. Suh, "Measurement of erythrocyte aggregation in a microchip stirring system by light transmission," Clinical Hemorheology and Microcirculation 41(3), 197-207 (2009).

15. S. Shin, J. Hou, J. Suh, and M. Singh, "Validation and application of a microfluidic ektacytometer (RheoScan-D) in measuring erythrocyte deformability," Clinical Hemorheology and Microcirculation 37(4), 319-28 (2007).

16. A. Abay, G. Simionato, R. Chachanidze, A. Bogdanova, L. Hertz, P. Bianchi, E. van den Akker, M. von Lindern, M. Leonetti, G. Minetti, C. Wagner, and L. Kaestner, "Glutaraldehyde - a subtle tool in the investigation of healthy and pathologic red blood cells," Frontiers in Physiology 10, 514 (2019).

17. G. Griffiths, "Fixation for fine structure preservation and immucytochemistry", Chapter in Fine Structure Immunocytochemistry, Springer, 26- 89 (1993).

18. N. Nemeth, F. Kiss, and K. Miszti-Blasius, "Interpretation of osmotic gradient ektacytometry (osmoscan) data: a comparative study for methodological standards," Scandinavian Journal of Clinical and Laboratory Investigation, 75(3), 213- $222(2015)$ 
19. Y. Liang, Y. Xiang, J. Lamstein, A. Bezryadina, and Z. Chen, "Cell deformation and assessment with tunable "tugof-war" optical tweezers," Conference on Lasers and Electro-Optics, AM1I.4 (2019).

20. R. Huisjes, A. Bogdanova, W. W. van Solinge, R. M. Schiffelers, L. Kaestner, R. van Wijk, "Squeezing for life properties of red blood cell deformability," Frontiers in Physiology 9, 514 (2018).

21. P. Snabre, M. Bitbol, and P. Mills, "Cell disaggregation behavior in shear flow," Biophysical Journal, 51(5), 795807 (1987).

22. M. Ju, S. S. Ye, H. T. Low, J. Zhang, P. Cabrales, H. L. Leo, and S. Kim, "Effect of deformability difference between two erythrocytes on their aggregation," Physical Biology 10(3), 036001 (2013).

23. S. Xue, B. Lee, and S. Shin. "Disaggregating shear stress: The roles of cell deformability and fibrinogen concentration," Clinical Hemorheology and Microcirculation 55(2), 231-240 (2012). 